\title{
DNA methylation contributes to silencing the expression of linc 00086 in gastric cancer
}

\author{
YANG YANG $^{1 *}$, YULONG LI $^{2 *}$, ZHENGHAO ZHAO $^{3}$, RUIFANG SUN $^{4}$, QIUYU JIANG ${ }^{3}$, LINGYU ZHAO $^{3}$,

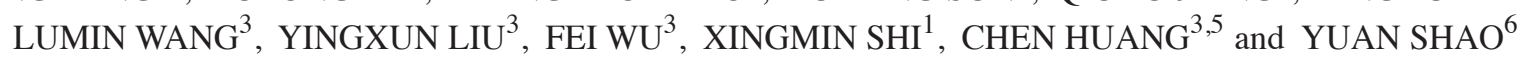 \\ ${ }^{1}$ School of Public Health, Xi'an Jiaotong University Health Science Center, Xi'an, Shaanxi 710061; ${ }^{2}$ Department of \\ Gastroenterology, Shaanxi Provincial People's Hospital, Xi'an, Shaanxi 710068; ${ }^{3}$ Key Laboratory of Environment and \\ Genes Related to Diseases, Xi'an Jiaotong University, Ministry of Education of China; ${ }^{4}$ Department of Pathology, \\ School of Basic Medical Sciences, Xi'an Jiaotong University Health Science Center, Xi'an, Shaanxi 710061; \\ ${ }^{5}$ Key Laboratory of Shaanxi Province for Craniofacial Precision Medicine Research, College of Stomatology, \\ Xi'an Jiaotong University, Xi'an, Shaanxi 710004; ${ }^{6}$ Department of Otorhinolaryngology, \\ First Affiliated Hospital of Medicine College of Xi'an Jiaotong University, Xi'an, Shaanxi 710061, P.R. China
}

Received April 14, 2017; Accepted September 7, 2017

DOI: $10.3892 / \mathrm{ol} .2018 .8868$

\begin{abstract}
Previous evidence has revealed that long non-coding RNAs serve important functions in numerous types of cancer when dysregulated, including in gastric cancer (GC). In the present study, reverse transcription-quantitative polymerase chain reaction (RT-qPCR) analysis was used to detect the expression of small integral membrane protein 10 like 2A (linc00086) in GC tissues and non-cancerous tissues, and the expression of linc00086 in GC cell lines was analyzed. A RT-qPCR assay was used to assess linc00086 expression levels in $\mathrm{GC}$ cell lines following treatment with 5-Aza-2'-deoxycytidine (5-aza-dC), which is a DNA methyltransferase inhibitor. Small interfering RNA was used to silence the expression of methyl- $\mathrm{CpG}$ binding protein 2 (MeCP2), and then the expression of linc00086 was detected. Linc00086 expression was revealed to be downregulated in GC tissues and GC cell lines. Furthermore, it was revealed that 5-aza-dC induced linc00086 expression in SGC-7901 and MKN45 cells, and analysis of CpG methylation by bisulfite
\end{abstract}

Correspondence to: Dr Chen Huang, Key Laboratory of Environment and Genes Related to Diseases, Xi'an Jiaotong University, Ministry of Education of China, 76 Yanta West Road, Xi'an, Shaanxi 710061, P.R. China

E-mail: hchen@mail.xjtu.edu.cn

Dr Yuan Shao, Department of Otorhinolaryngology, First Affiliated Hospital of Medicine College of Xi'an Jiaotong University, 277 Yanta Western Road, Xi'an, Shaanxi 710061, P.R. China

E-mail: shaxiaoying1976@163.com

*Contributed equally

Key words: DNA methylation, small integral membrane protein 10 like $2 \mathrm{~A}$, gastric cancer sequencing-polymerase chain reaction demonstrated that DNA methylation may regulate the expression of linc00086. MeCP2 is involved in gene regulation by binding to methylated promoters, and it was revealed that the knockdown of the expression of $\mathrm{MeCP} 2$ resulted in a higher expression of linc00086. The present study revealed that DNA methylation regulate the expression of linc00086 in human GC cell lines.

\section{Introduction}

Gastric cancer (GC) remains one of the most common types of cancer globally (1). Gastric carcinogenesis is a multistep and multifactorial process (2) and may be affected by various factors that participate in each carcinogenic step, including the activation of oncogenes, the inactivation of tumor suppressor genes (3) and the effects of epigenetic modification (4). Each of these factors may affect the occurrence and development of GC. Investigating the molecular regulation of GC development is essential for diagnosis and treatment. Methyl-CpG binding protein 2 (MeCP2) is a methylated binding protein, which may bind to methylated $\mathrm{CpG}$ islands and inhibit the transcription of genes (5). MeCP2 may also serve an oncogenic function in GC (6). Long noncoding RNAs (lncRNAs) are a class of noncoding RNA which are $>200$ nucleotides long (7). Increasingly, studies have revealed that the deregulation of lncRNAs are involved in a variety of human diseases and serve important functions in cell proliferation, apoptosis, metastasis and invasion (8-11). Small integral membrane protein 10 like 2A (linc00086), located at the $\mathrm{X}$ chromosome, is required for the tumor protein p53 transcriptional response (12).

In the present study, the expression of linc00086 was revealed to be downregulated in GC. Bioinformatics analyses revealed that the linc00086 gene has $\mathrm{CpG}$ islands. In order to further investigate whether linc00086 methylation was responsible for the downregulated expression of linc00086 in GC, in the present study GC cells were treated with 5-Aza-2'-deoxycytidine (5-aza-dC) and it was demonstrated 
that linc00086 expression was upregulated. Bisulfite sequencing-polymerase chain reaction (PCR) was used to detect the methylation effect on linc00086, and analysis of $\mathrm{CpG}$ methylation by bisulfite sequencing-PCR demonstrated that DNA methylation may regulate the expression of linc00086. As $\mathrm{MeCP} 2$ is involved in gene regulation by binding to methylated promoters, the expression of MeCP2 was silenced in order to detect the expression of linc00086. The results revealed that silencing the expression of $\mathrm{MeCP} 2$ may promote the expression of linc00086. These results suggested that DNA methylation may contribute to silencing the expression of linc00086 in GC.

\section{Materials and methods}

Human tissue samples and cell lines. The GC cell lines SGC-7901, MKN45, BGC-823, AGS and the normal gastric cell line GES-1 were sourced from the Key Laboratory of Environment and Genes Related to Diseases, Xi'an Jiaotong University, Ministry of Education of China (Xi'an, China). These cells were cultured in RPMI-1640 medium (Gibco; Thermo Fisher Scientific, Inc., Waltham, MA, USA) supplemented with $10 \%$ fetal bovine serum (Gibco; Thermo Fisher Scientific, Inc.) at $37^{\circ} \mathrm{C}$ in a humidified chamber with $5 \% \mathrm{CO}_{2}$. A total of $20 \mathrm{GC}$ tissues (all males; mean age, 59.5 years; age range, 43-74 years) and the matched normal tissues were sourced from the First Affiliated Hospital of Xi'an Jiaotong University College of Medicine (Xi'an, China). No radiotherapy or chemotherapy was conducted prior to surgery. The present study was approved by the Medical Ethical Committee of the College of Medicine, Xi'an Jiaotong University. Written informed consent was provided by all patients.

RNA extraction and reverse transcription-quantitative $(R T-q) P C R$ analysis. RNA was extracted using TRIzol ${ }^{\circledR}$ reagent (Invitrogen; Thermo Fisher Scientific, Inc.) according to the manufacturer's protocol. Complementary DNA synthesis was performed using the Prime-Script RT reagent kit (Takara Biotechnology Co., Ltd., Dalian, China) according to the manufacturer's protocol. qPCR was performed by SYBR Green PCR kit (Takara Biotechnology Co., Ltd.) to analyze the expression levels of the genes. $\beta$-actin was used as an endogenous control Thermocycling conditions were as follows: Initial denaturation at $95^{\circ} \mathrm{C}$ for $30 \mathrm{sec}$, followed by 40 cycles at $95^{\circ} \mathrm{C}$ for $5 \mathrm{sec}$ and at $60^{\circ} \mathrm{C}$ for $30 \mathrm{sec}$. The relative expression of genes was calculated using the $2-\Delta \Delta \mathrm{Cq}$ method (13). The primers used are listed in Table I.

5-aza-dC treatment. SGC-7901 and MKN45 cells were treated with the DNA methyltransferase inhibitor, 5-aza-dC (Sigma-Aldrich; Merck KGaA, Darmstadt, Germany) at differing concentrations $(0.0,2.0,4.0,6.0$ and $8.0 \mu \mathrm{M})$ for $48 \mathrm{~h}$ $37^{\circ} \mathrm{C}$. Then the RNA was isolated from SGC-7901 or MKN45 cells using TRIzol (Invitrogen; Thermo Fisher Scientific, Inc.) according to the manufacturer's protocol. RT-qPCR was performed to detect the expression of linc00086 as aforementioned. The primers used are listed in Table I.

Bisulfite sequencing PCR. SGC-7901 and MKN45 cells were treated with $2.0 \mu \mathrm{M}$ 5-aza-dCor equal amounts of DMSO as a control for $48 \mathrm{~h}$ at $37^{\circ} \mathrm{C}$, and DNA was extracted and modified
Table I. Primer sequences.

Name of primer

Sequence (5'-3')

Linc00086, forward

Linc00086, reverse

Linc00086 CPG

islands, forward

Linc00086 CPG

islands, reverse

Linc00086, small integral membrane protein 10 like 2A.

using the bisulfite reaction according to the manufacturer's protocol of the Qiagen EpiTect ${ }^{\circledR}$ Bisulfite kit (Qiagen, Inc., Valencia, CA, USA). Primer sequences are listed in Table I. PCR used 2xTaq MasterMix (Dye; CoWin Biosciences Co., Ltd., Jiangsu, China). The total reaction volume was $50 \mu 1$ in a mixture containing $0.4 \mu \mathrm{g}$ DNA, and the thermocycler conditions were as follows: Pre-denaturation at $94^{\circ} \mathrm{C}$ for $2 \mathrm{~min}$, followed by 35 cycles of denaturation at $94^{\circ} \mathrm{C}$ for 30 seconds, annealing at $59^{\circ} \mathrm{C}$ for 30 seconds, extension at $72^{\circ} \mathrm{C}$ for 30 seconds, with a final extension step at $72^{\circ} \mathrm{C}$ for $2 \mathrm{~min}$. The PCR products of bisulfite-modified DNA of SGC-7901 cells were purified and cloned into a T-vector (Takara Biotechnology Co., Ltd.), then sequenced by Sangon Biotech Co., Ltd. (Shanghai, China). The methylation level was analyzed using the Quantification Tool for Methylation Analysis (14).

Small interfering RNA (siRNA) transfection. A total of $5 \times 10^{5}$ SGC-7901 or MKN45 cells were seeded for $24 \mathrm{~h}$ prior to transfection. SiRNAs against MeCP2 (si-MeCP2) with sequences as follows: si-MeCP2-S, GCUUAAGCAAAGGAAAUCUTT and si-MeCP2-A, AGAUUUCCUUUGCUUAAGCTT (15) and its respective siRNA-negative controls (si-control) with sequences as follows: siRNA-ctrl-S UUCUCCGAACGU GUCACGUTT and siRNA-ctrl-A, ACGUGACACGUUCGG AGAATT (Shanghai GenePharma Co., Ltd., Shanghai, China) were transfected into the cells using Lipofectamine 2000 (Invitrogen) according to the manufacturer's protocol. RNA was extracted $48 \mathrm{~h}$ post-transfection, and then RT-qPCR was used to detect the expression of MeCP2 and linc00086 as aforementioned.

Statistical analysis. All statistical analyses were performed using SPSS 13.0 software (SPSS, Inc., Chicago, IL, USA). Student's t-test was used to analyze the data. $\mathrm{P}<0.05$ was considered to indicate a statistically significant difference.

\section{Results}

Linc00086 is downregulated in GC tissue and cell lines. RT-qPCR was performed to detect the expression levels of linc00086 in GC tissues and cell lines. The results revealed that among the 20 paired samples, 17 of them (85\%) exhibited a lower expression of linc00086 in GC tissues compared with the matched non-tumor gastric tissues (Fig. 1A). Furthermore, when comparing the expression levels of linc00086 in AGS, 

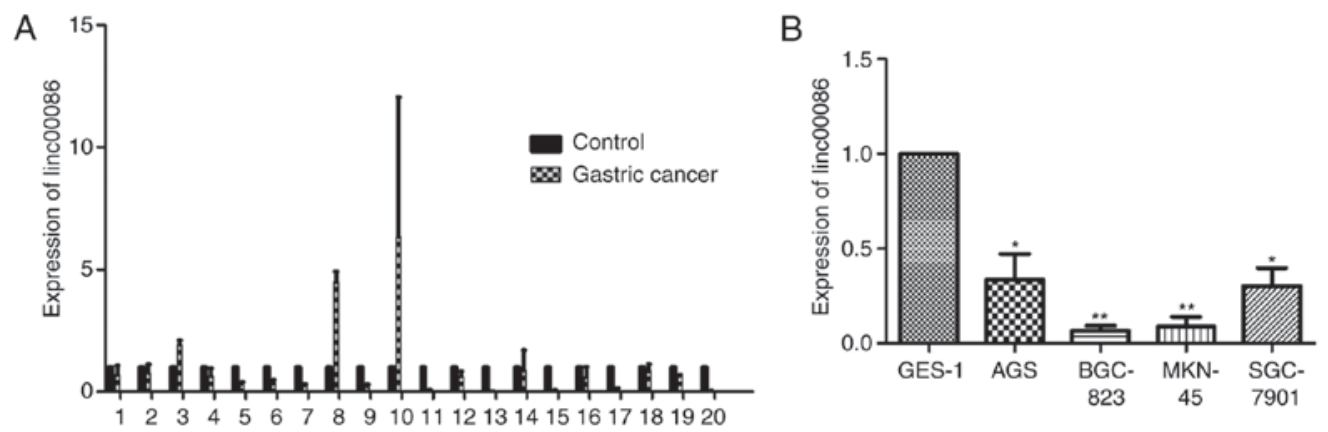

Figure 1. Linc00086 is significantly downregulated in GC tissue samples and cell lines. (A) The expression of linc00086 was lower in GC tissues than in normal tissues in 17/20 paired samples (85\%). (B) The expression of linc00086 was lower in gastric cell lines (AGS, BGC-823, MKN-45 and SGC-7901) than in a normal human gastric epithelial cell line (GES-1). ${ }^{* *} \mathrm{P}<0.01$ and " $\mathrm{P}<0.05$, vs. GES-1. Linc00086, small integral membrane protein 10 like $2 \mathrm{~A}$; GC, gastric cancer.
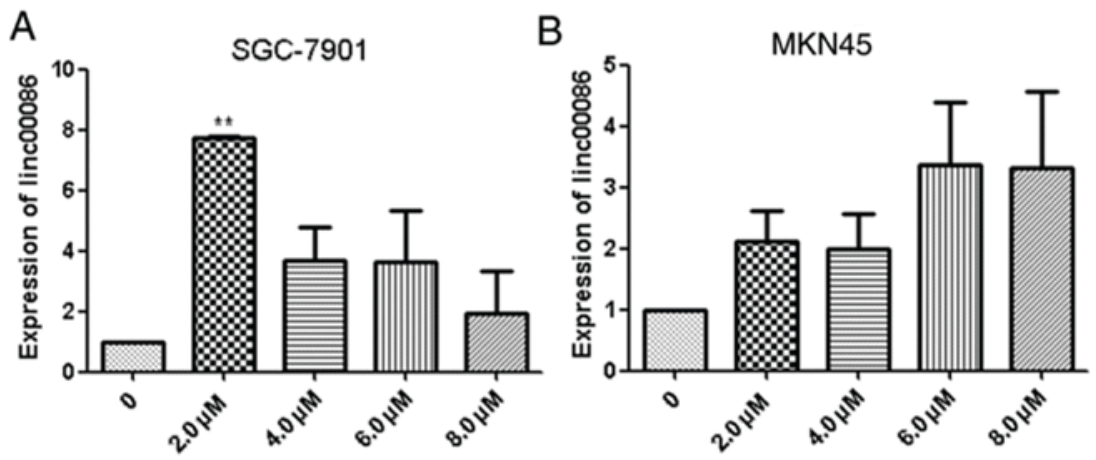

Figure 2.5-aza-dC treatment induces linc00086 expression. Reverse transcription-quantitative polymerase chain reaction was performed to detect linc00086 expression levels in (A) SGC-7901 cells treated with 5-aza-dC (0.0, 2.0, 4.0, 6.0and $88.0 \mu \mathrm{M})$ for $48 \mathrm{~h}$ and (B) MKN45 cells treated with 5-aza-dC (0.0, 2.0, 4.0.0, 6.0 and $8.0 \mu \mathrm{M})$ for $48 \mathrm{~h} .{ }^{* *} \mathrm{P}<0.01$ vs. the $0 \mu \mathrm{M}$ treatment group. 5-aza-dC, 5-aza-2'-deoxycytidine; linc00086, small integral membrane protein 10 like $2 \mathrm{~A}$.

BGC-823, MKN-45 and SGC-7901cells with GES-1 cells, the expression level of linc00086 was significantly downregulated in the GC cell lines, compared with GES-1 cells (Fig. 1B). The results of the present study suggest that linc00086 was downregulated in GC tissue and cell lines.

5-aza-dC treatment may increase the expression of linc00086. The potential for DNA methylation to contribute to the silencing of the expression of linc00086 in GC was investigated. SGC-7901 and MKN-45 cells were treated with 0.0,2.0, 4.0, 6.0 and $8.0 \mu \mathrm{M} 5$-aza-dC for $48 \mathrm{~h}$. The results revealed that treatment with 5 -aza-dC may induce the expression of linc00086 (Fig. 2A and B). It was revealed that treatment with $2.0 \mu \mathrm{M} 5$-aza-dC for $48 \mathrm{~h}$ was the optimal concentration.

Methylation levels declined following treatment with 5-aza- $d C$. SGC-7901 and MKN45 cells were treated with $2.0 \mu \mathrm{M} 5$-aza-dCor control, and then DNA was extracted. DNA was modified using the bisulfite reaction. Primers of bisulfite sequencing-PCR and bisulfite modified DNA were used to determine the interest region sequences of the linc00086 CPG islands using PCR (Fig. 3A). Next, the PCR products of bisulfite-modified DNA of SGC-7901 were cloned into a T-vector and sequenced. The methylation level of the control group was $20 \%$ and the methylation level of the 5-aza-dC treatment group was $10 \%$ in the SGC-7901 cells. The methylation level of 5-aza-dC treatment group was decreased compared with the control group (Fig. 3B). The results suggested that the methylation level declined following treatment with 5-aza-dC.

Silencing MeCP2 may induce the expression of linc00086. SGC-7901 andMKN45 cells were transfected with si-MeCP2 or si-control. RNA was extracted $48 \mathrm{~h}$ post-transfection, and then expression of MeCP2 and linc00086 was detected. It was revealed that si-MeCP2 may substantially reduce the expression of MeCP2 (Fig. 4A and B), and si-MeCP2 may increase the expression of linc00086 in SGC-7901 and MKN45 cells (Fig. 4C and D). The results of the present study further confirm that the low expression of linc00086 in GC is regulated by DNA methylation.

\section{Discussion}

DNA methylation is an important type of epigenetic modification, and has been reported to be involved in tumorigenesis. Previous studies have identified that DNA methylation changes may result in aberrant gene expression in cancer $(16,17)$, and a number of them may be an early molecular marker $(18,19)$. A number of differing factors may silence a number of tumor suppressors due to DNA methylation, including miR-122 (20), miR-219-2-3p (21), miR-203 (22), and lncRNAs SRHC (23) and maternally expressed 3 (non-protein coding) (24). The 
A SGC-7901 MKN-45 Marker Control 5-aza-dC Control 5-aza-dC

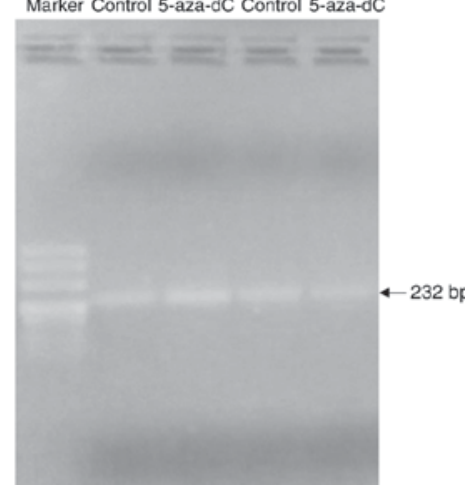

B

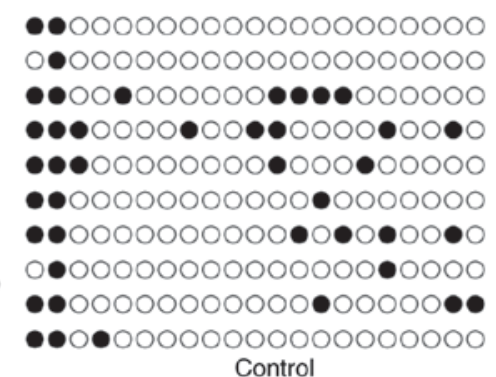

SGC-7901

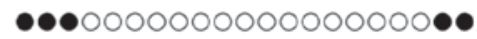
○०0000000000000000000 - 0000000000000000000 000000000000000000000 - 00000000000000000 $000000000000000000 \bullet 00$

- 000000000000000000

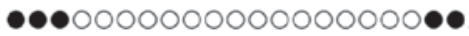
000000000000000000000 5-aza-dC

Figure 3. Methylation declines following treatment with 5-aza-dC in SGC-7901 cells. (A) The size of the amplified PCR products of bisulfite-treated DNA (the DNA was extracted from SGC-7901 and MKN45 cells treated with $2.0 \mu \mathrm{M} 5$-aza-dC or control). (B) The methylation level of linc00086 in the control group and 5-aza-dC treated group in SGC-7901 cells were analyzed following the cloning of PCR products into the T-vector sequence. 5-aza-dC, 5-aza-2'-deoxycytidine; PCR, polymerase chain reaction; linc00086, small integral membrane protein 10 like 2A.

A

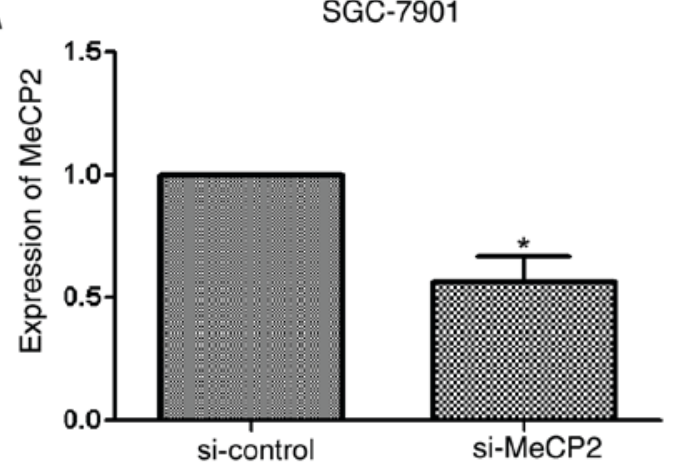

C

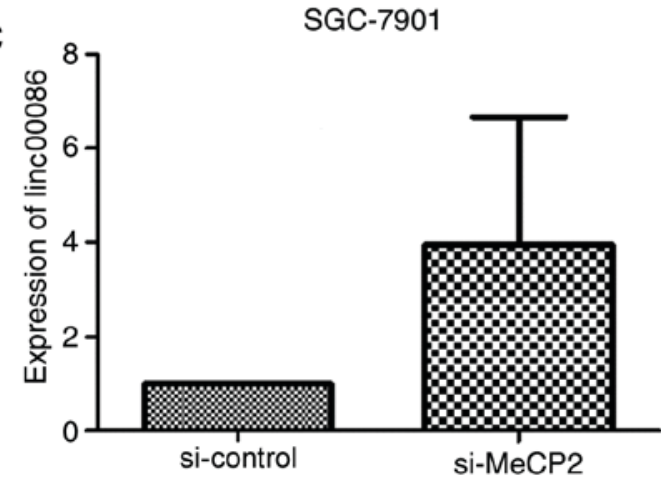

B

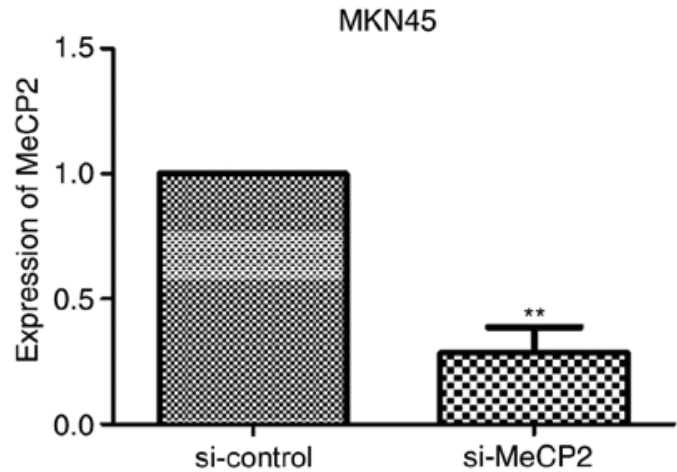

D

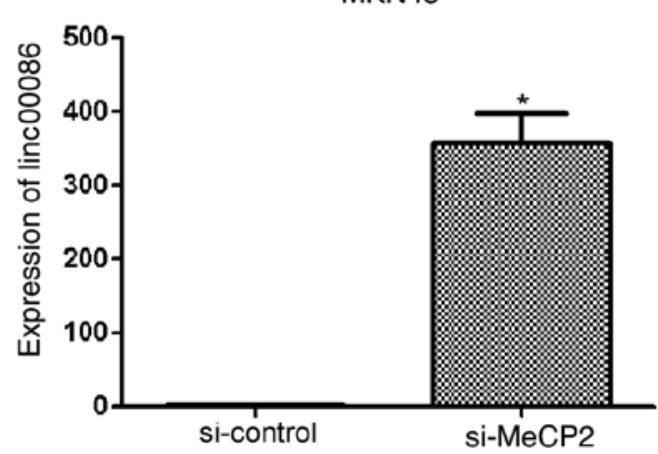

Figure 4. Silencing MeCP2 may induce the expression of linc00086. RT-qPCR was performed to detect the expression of MeCP2 in (A) SGC-7901 and (B) MKN45 cells transfected with si-MeCP2 or si-control for $48 \mathrm{~h}$. Reverse transcription-quantitative polymerase chain reaction was used to detect the expression of linc00086 in (C) SGC-7901 and (D) MKN45 cells transfected with si-MeCP2 or si-control for $48 \mathrm{~h}$. ${ }^{* *} \mathrm{P}<0.01$ and ${ }^{*} \mathrm{P}<0.05$ vs. the si-control group. MeCP2, methyl-CpG binding protein 2; linc00086, small integral membrane protein 10 like 2A; si-MeCP2, small interfering RNA against MeCP2; si-control, small interfering RNA-negative control.

focus of the present study was on the silencing of linc00086 by DNA methylation in GC.

Previously, studies have revealed the participation of lncRNAs in various biological processes. Furthermore, a number of lncRNAs are detectable as biomarkers in the diagnosis of certain types of cancer, including the following lncRNAs: Urothelial cancer associated 1 for GC (25), POU class 3 homeobox 3 for esophageal squamous cell carcinoma (26), PVT1 oncogene (non-protein coding) for cervical cancer (27) and CCDC26 long non-coding RNA for pancreatic cancer (28).
The present study revealed that linc00086 expression was lower in GC tissues compared with normal tissues. Accumulating evidence has revealed that a number of lncRNAs were aberrant in cancer (29). The association between linc00086 expression and the survival period of patients with GC will be further studied through the use of databases of a suitable sample size. Furthermore, the present study revealed that the aberrant expression of linc00086 may be regulated by DNA methylation in GC cell lines. DNA methylation and histone modification are the two major types 
of epigenetic modification. In the present study, bisulfite sequencing-PCR was used to identify the DNA methylation of linc00086 in SGC-7901 cells in order to explore the reason behind the low expression of linc00086. The results of the present study have demonstrated that DNA methylation may be one of the reasons for the silenced expression of linc00086 in GC. MeCP2 belongs to the family of methyl-CpG-binding proteins that regulate gene expression by DNA methylation (30). MeCP2 has previously emerged as an important oncogene in a multitude of types of cancer, and is involved in cancer progression. It has been reported that $\mathrm{MeCP} 2$ expression is increased in hepatocellular carcinoma and promotes the proliferation of human hepatocellular carcinoma HepG2 cells via the activation of extracellular signal-regulated kinase 1/2 signaling pathways (31). MeCP2 was overexpressed in GC and serves an important function in gastric carcinogenesis (15). MeCP2 may regulate gene expression by binding methylated $\mathrm{CpG}$ islands (32). The present study revealed that silencing MeCP2 may induce the expression of linc00086, which may suggest that the downregulated expression of linc00086 in GC is associated with DNA methylation. The results of the present study identified that the aberrant expression of linc00086 may be regulated by DNA methylation.

\section{Acknowledgements}

Not applicable.

\section{Funding}

The present study was funded by Research Support Project of New Teacher of Xi'an Jiaotong University (grant no. YX1K078).

\section{Availability of data and materials}

The datasets used and/or analyzed during the current study are available from the corresponding author on reasonable request.

\section{Authors' contributions}

$\mathrm{CH}$ and YS conceived the study. RS, LZ, LW and XS collected the cancer tissues. YY, ZZ, QJ, YLL, YXL and FW performed the experiments. $\mathrm{CH}$ and $\mathrm{YY}$ calculated the data. YXL and YY wrote the paper.

\section{Ethics approval and consent to participate}

The research protocol was approved by the Medical Ethical Committee of the College of Medicine, Xi'an Jiaotong University. Written informed consent was provided by all patients.

\section{Consent for publication}

Written informed consent was obtained from all patients.

\section{Competing interests}

The authors declare that they have no competing interests.

\section{References}

1. Bertuccio P, Chatenoud L, Levi F, Praud D, Ferlay J, Negri E, Malvezzi M and La Vecchia C: Recent patterns in gastric cancer: A global overview. Int J Cancer 125: 666-673, 2009.

2. Correa P: Human gastric carcinogenesis: A multistep and multifactorial process-first American cancer society award lecture on cancer epidemiology and prevention. Cancer Res 52: 6735-6740, 1992.

3. Tahara E, Yasui $\mathrm{W}$ and Yokozaki H: Genetic alterations in stomach cancer. Nihon Geka Gakkai zasshi 97: 252-256, 1996 (In Japanese)

4. Patel TN, Roy S and Ravi R: Gastric cancer and related epigenetic alterations. Ecancermedicalscience 11: 714, 2017.

5. Wakefield RI, Smith BO, Nan X, Free A, Soteriou A, Uhrin D, Bird AP and Barlow PN: The solution structure of the domain from MeCP2 that binds to methylated DNA. J Mol Biol 291: 1055-1065, 1999

6. Zhao L, Liu Y, Tong D, Qin Y, Yang J, Xue M, Du N, Liu L, Guo B, Hou N, et al: $\mathrm{MeCP} 2$ promotes gastric cancer progression through regulating FOXF1/Wnt5a/ $\beta$-Catenin and MYOD1/Caspase-3 signaling pathways. EBioMedicine 16: 87-100, 2017.

7. Rinn JL and Chang HY: Genome regulation by long noncoding RNAs. Annu Rev Biochem 81: 145-166, 2012.

8. Wang F, Yuan JH, Wang SB, Yang F, Yuan SX, Ye C, Yang N, Zhou WP, Li WL, Li W and Sun SH: Oncofetal long noncoding RNA PVT1 promotes proliferation and stem cell-like property of hepatocellular carcinoma cells by stabilizing NOP2. Hepatology 60: 1278-1290, 2014.

9. Wu ZJ, Li Y, Wu YZ, Wang Y, Nian WQ, Wang LL, Li LC, Luo HL and Wang DL: Long non-coding RNA CCAT2 promotes the breast cancer growth and metastasis by regulating TGF- $\beta$ signaling pathway. Eur Rev Med Pharmacol Sci 21: 706-714, 2017.

10. Wang D, Wang D, Wang N, Long $Z$ and Ren X: Long Non-Coding RNA BANCR promotes endometrial cancer cell proliferation and invasion by regulating MMP2 and MMP1 via ERK/MAPK signaling pathway. Cell Physiol Biochem 40: 644-656, 2016

11. Wang J, Zhang X, Shi J, Cao P, Wan M, Zhang Q, Wang Y, Kridel SJ, Liu W, Xu J, et al: Fatty acid synthase is a primary target of MiR-15a and MiR-16-1 in breast cancer. Oncotarget 7: 78566-78576, 2016.

12. Leveille N, Melo CA, Rooijers K, Díaz-Lagares A, Melo SA, Korkmaz G, Lopes R, Akbari Moqadam F, Maia AR, Wijchers PJ, et al: Genome-wide profiling of p53-regulated enhancer RNAs uncovers a subset of enhancers controlled by a lncRNA. Nat Commun 6: 6520, 2015.

13. Livak KJ and Schmittgen TD: Analysis of relative gene expression data using real-time quantitative PCR and the 2(-Delta Delta $\mathrm{C}(\mathrm{T})$ ) method. Methods 25: 402-408, 2001.

14. Kumaki Y, Oda M and Okano M: QUMA: Quantification tool for methylation analysis. Nucleic Acids Res 36: W170-W175, 2008.

15. Tong D, Zhao L, He K, Sun H, Cai D, Ni L, Sun R, Chang S, Song T and Huang C: MECP2 promotes the growth of gastric cancer cells by suppressing miR-338-mediated antiproliferative effect. Oncotarget 7: 34845-34859, 2016.

16. Nordstrom L, Andersson E, Kuci V, Gustavsson E, Holm K, Ringnér M, Guldberg P and Ek S: DNA methylation and histone modifications regulate SOX11 expression in lymphoid and solid cancer cells. BMC Cancer 15: 273, 2015.

17. Tian Y, Wei W, Li L and Yang R: Down-Regulation of miR-148a Promotes Metastasis by DNA Methylation and is associated with prognosis of skin cancer by targeting TGIF2. Med Sci Monit 21: 3798-3805, 2015.

18. Liu K, Zhang Y, Zhang C, Zhang Q, Li J, Xiao F, Li Y, Zhang R, Dou D, Liang J, et al: Methylation of S100A8 is a promising diagnosis and prognostic marker in hepatocellular carcinoma. Oncotarget 7: 56798-56810, 2016.

19. Pimson C, Ekalaksananan T, Pientong C, Promthet S, Putthanachote N, Suwanrungruang K and Wiangnon S: Aberrant methylation of PCDH10 and RASSF1A genes in blood samples for non-invasive diagnosis and prognostic assessment of gastric cancer. PeerJ 4: e2112, 2016.

20. Xing TJ, Xu HT, Yu WQ and Jiang DF: Methylation regulation of liver-specific microRNA-122 expression and its effects on the proliferation and apoptosis of hepatocellular carcinoma cells. Genet Mol Res 12: 3588-3597, 2013. 
21. Lei H, Zou D, Li Z, Luo M, Dong L, Wang B, Yin H, Ma Y, Liu C, Wang F, et al: MicroRNA-219-2-3p functions as a tumor suppressor in gastric cancer and is regulated by DNA methylation. PLoS One 8: e60369, 2013.

22. Noguchi S, Mori T, Nakagawa T, Itamoto K, Haraguchi $T$ and Mizuno T: DNA methylation contributes toward silencing of antioncogenic microRNA-203 in human and canine melanoma cells. Melanoma Res 25: 390-398, 2015

23. Zheng H, Yang S, Yang Y, Yuan SX, Wu FQ, Wang LL, Yan HL, Sun SH and Zhou WP: Epigenetically silenced long noncoding-SRHC promotes proliferation of hepatocellular carcinoma. J Cancer Res Clin Oncol 141: 1195-1203, 2015.

24. Sun M, Xia R, Jin F, Xu T, Liu Z, De W and Liu X: Downregulated long noncoding RNA MEG3 is associated with poor prognosis and promotes cell proliferation in gastric cancer. Tumour Biol 35: 1065-1073, 2014

25. Gao J, Cao R and Mu H: Long non-coding RNA UCA1 may be a novel diagnostic and predictive biomarker in plasma for early gastric cancer. Int J Clin Exp Pathol 8: 12936-12942, 2015.

26. Tong YS, Wang XW, Zhou XL, Liu ZH, Yang TX, Shi WH, Xie HW, Lv J, Wu QQ and Cao XF: Identification of the long non-coding RNA POU3F3 in plasma as a novel biomarker for diagnosis of esophageal squamous cell carcinoma. Mol Cancer 14: 3, 2015.
27. Yang JP, Yang XJ, Xiao L and Wang Y: Long noncoding RNA PVT1 as a novel serum biomarker for detection of cervical cancer. Eur Rev Med Pharmacol Sci 20: 3980-3986, 2016.

28. Peng W and Jiang A: Long noncoding RNA CCDC26 as a potential predictor biomarker contributes to tumorigenesis in pancreatic cancer. Biomed Pharmacother 83: 712-717, 2016.

29. Gibb EA, Vucic EA, Enfield KS, Stewart GL, Lonergan KM, Kennett JY, Becker-Santos DD, MacAulay CE, Lam S, Brown CJ and Lam WL: Human cancer long non-coding RNA transcriptomes. PLoS One 6: e25915, 2011.

30. Lyu JW, Yuan B, Cheng TL, Qiu ZL and Zhou WH: Reciprocal regulation of autism-related genes MeCP2 and PTEN via microRNAs. Sci Rep 6: 20392, 2016.

31. Zhao LY, Zhang J, Guo B, Yang J, Han J, Zhao XG, Wang XF, Liu LY, Li ZF, Song TS and Huang C: MECP2 promotes cell proliferation by activating ERK1/2 and inhibiting p38 activity in human hepatocellular carcinoma HEPG2 cells. Cell Mol Biol (Noisy-le-grand) Suppl 59: OL1876-OL1881, 2013.

32. Xu M, Bian S, Li J, He J, Chen H, Ge L, Jiao Z, Zhang Y, Peng W, Du F, et al: MeCP2 suppresses LIN28A expression via binding to its methylated-CpG islands in pancreatic cancer cells. Oncotarget 7: 14476-14485, 2016. 